\title{
Sensores de presión basados en interferómetros de fibra de cristal fotónico
}

\author{
Ángel Sanz, Juan Carlos Martín \\ Grupo de Tecnología Óptica Láser (TOL) \\ Instituto de Investigación en Ingeniería de Aragón (I3A) \\ Universidad de Zaragoza, Mariano Esquillor s/n, 50018, Zaragoza, Spain. \\ Tel.+34-976762707, e-mail: angel_sf@unizar.es
}

\begin{abstract}
Analizamos los interferómetros modales basados en fibra de cristal fotónico como sensores de presión. Bajo presión estática observamos cambios en la transmisión del interferómetro, con sensibilidad de $\sim 0.2 \%$ por $\mathrm{g} / \mathrm{cm}$ y comportamiento lineal para cargas inferiores a 30-40 g. Para presiones oscilantes el sistema muestra buena linealidad y comportamiento resonante.
\end{abstract}

\section{Introducción}

Es común el uso de fibras ópticas como elemento base de sensores de magnitudes físicas como temperatura, elongación y presión $[1,2]$. En nuestro trabajo analizamos los cambios de potencia transmitida en un interferómetro de fibra de cristal fotónico al ejercer sobre ella una fuerza transversal. $\mathrm{Su}$ utilidad como sensor de presión requiere una respuesta lineal, reproducible y con suficiente sensibilidad a pequeñas variaciones de presión.

\section{Montaje experimental}

El sensor de presión se basa en un interferómetro formado por fibra de cristal fotónico de $4.5 \mathrm{~cm}$ de longitud soldada por los extremos a fibra estándar monomodo.

Utilizamos dos fuentes de luz (SLED o láser sintonizable), detectada tras atravesar la fibra por un OSA (Optical Spectrum Analyzer) o por un fotodiodo PIN conectado al osciloscopio. El montaje depende del tipo de medidas a realizar: si son en modo estático (Fig. 1.a) o dinámico (Fig. 1.b), y de si estudiamos el espectro de 1470-1640 $\mathrm{nm}$ o medimos con una $\lambda$ en particular.

Para ejercer la fuerza estática utilizamos una varilla que presiona sobre $3 \mathrm{~mm}$ de longitud de la fibra, o un apoyo de $1 \mathrm{~cm}$ de longitud sobre el que añadir peso depositando agua. Para producir fuerzas dinámicas utilizamos una varilla acoplada a la membrana de un altavoz apoyada sobre $1 \mathrm{~cm}$ de longitud de la fibra.

\section{Resultados}

\section{Presión estática}

La Fig. 2 muestra cómo se producen cambios en el espectro interferencial de la fibra al aumentar la presión sobre ésta: se observa un desplazamiento al rojo de las interferencias que separa los picos [3], y un aumento de contraste de tales interferencias [4]. A partir de estas variaciones en el espectro interferencial, se seleccionan las $\lambda$ 's cuya transmisión es más sensible al cambio de presión. En los mejores casos, se tiene una sensibilidad del $\sim 0.2 \%$ de la transmisión por $\mathrm{g} / \mathrm{cm}$ con un coeficiente de correlación lineal $\mathrm{R}^{2}>0.9$.

Por otro lado, la Fig. 3.a muestra la respuesta del dispositivo para dos $\lambda$ 's concretas. Analizando la potencia transmitida en función de la presión se comprueba la linealidad de las medidas, obteniéndose los valores de cambio de potencia en función de la carga apoyada que esperábamos obtener en comparación con el espectro interferencial para estas $\lambda$ 's en la Fig. 2. La respuesta lineal comienza a desparecer en algunos casos con cargas en torno a 20-30 g dependiendo de la $\lambda$ seleccionada, dándose cambios bruscos de potencia o saturación.

\section{Presión dinámica}

Comprobada la linealidad del sistema bajo fuerza estática, cabe preguntarse si también se da tal comportamiento cuando la fuerza depende del tiempo. Al variar sinusoidalmente la fuerza aplicada, vemos en la Fig. 3.b que la comparación entre amplitudes pico-pico de la señal de excitación del oscilador y la potencia detectada en el fotodiodo ofrece un comportamiento lineal para una frecuencia fija, que se comprueba para amplios rangos de frecuencia y también de amplitud hasta un punto en que comienza a saturar.

Por otro lado, en el osciloscopio comprobamos que la potencia transmitida oscila sinusoidalmente como lo hace la presión, pero la amplitud depende 
fuertemente de la frecuencia de las oscilaciones, como se ve en la Fig. 4.a. En particular, se observa en la respuesta la aparición de resonancias en las que la amplitud de la señal detectada aumenta claramente sin hacerlo la señal aplicada, como cabe esperar en un sistema oscilante forzado-amortiguado como es el caso. La Fig. 4.b muestra que además aparece un desfase entra las oscilaciones de excitación y transmisión que depende de la frecuencia. Asimismo, al producir estas oscilaciones con amplitud de excitación suficientemente grande para el sistema, aparecen armónicos de la frecuencia de excitación, señal de que la respuesta del sistema deja de ser lineal.

\section{Conclusiones}

Se han verificado los cambios de transmisión por variaciones de presión en un interferómetro basado en fibra de cristal fotónico. Se comprueba su linealidad y sensibilidad con cambios pequeños de presión estática y su capacidad como sensor. Para presiones dinámicas se comprueba la linealidad a una frecuencia dada y que la respuesta depende fuertemente de la frecuencia de vibración, lo que implica que para cuantificar las vibraciones es necesario calibrar en frecuencia el sensor.

\section{REFERENCIAS}

[1]. BOCK, W.J., EFTIMOV, T., MIKULIC, P., and CHEN, J. Novel Fiber Optic Sensor Based on In-Line Core-Cladding Intermodal Interferometer and Photonic Crystal Fiber. XIX IMEKO World Congress. Fundamental and Applied Metrology. Sep. 6-11, 2009, Lisbon, Portugal. ISBN 978-963-88410-0-1.

[2]. LIU, Z., WU, C., TSE, M.L.V., and TAM, H.Y. Fabrication, Characterization, and Sensing Applications of a High-Birefringence SuspendedCore Fiber. J. Light. Technol., vol. 32, no. 11, pp. 2113-2122, Jun. 2014.

[3]. URBAN, F., KADLEC, J., VLACH, R., and KUCHTA, R. Desing of a Pressure Sensor Based on Optical Fiber Bragg Grating Lateral Deformation. Sensors 2010, 10, 11212-11225. Available from doi: 10.3390/s101211212.

[4]. YU, X., BU, D., CHEN, X., ZHANG, J., and LIU, S. Lateral Stress Sensor Based on an In-Fiber MachZehnder Interferometer and Fourier Analysis. IEEE Photon. J., vol. 8, no. 2, 6801710, Apr. 2016. Available from: doi:10.1109/JPHOT.2016.2538958.

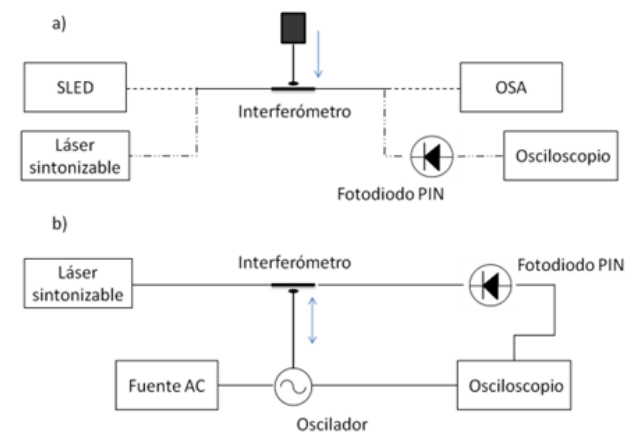

Fig. 1. Esquema de los montajes para medidas estáticas (a) y dinámicas (b).

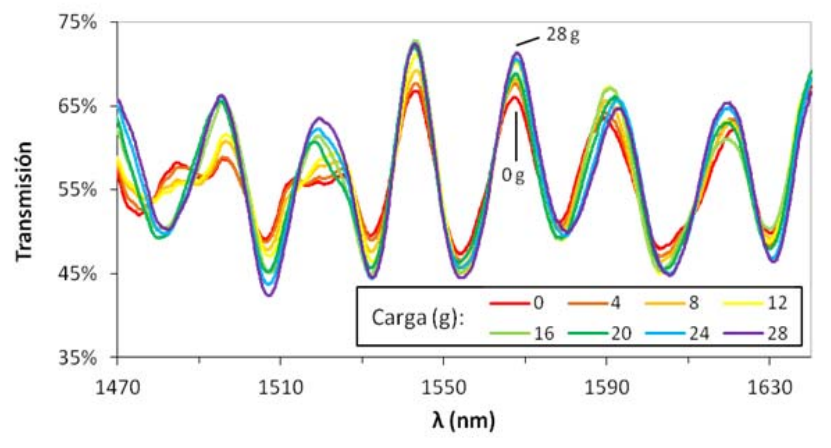

Fig. 2. Espectro interferencial de la transmisión en función de la carga sobre $3 \mathrm{~mm}$ de longitud.
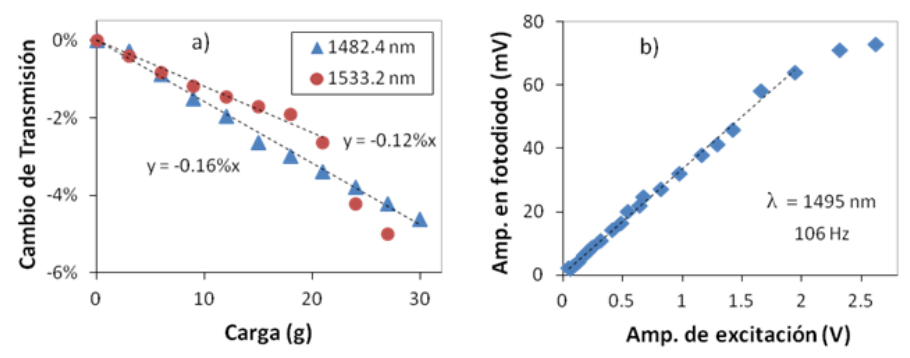

Fig. 3. Cambio de potencia transmitida bajo presión estática (a) y dinámica (b) sobre $1 \mathrm{~cm}$ de longitud.

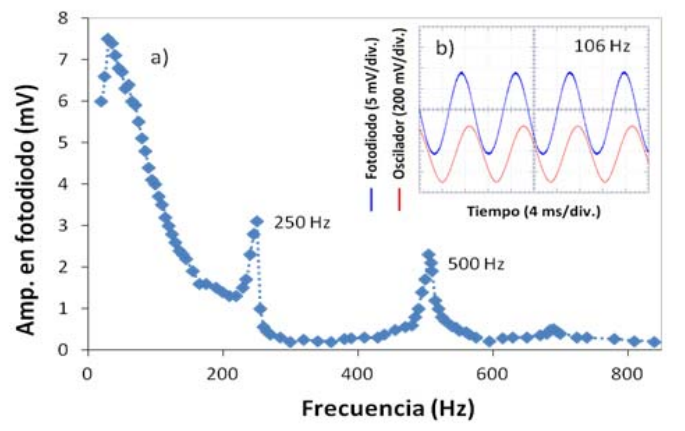

Fig. 4. (a) Amplitud de la potencia en función de la frecuencia y (b) desfase entre transmisión y excitación. 